\title{
Two Combined Alphabetic Optimality Criteria for Second Order Rotatable Designs Constructed Using Balanced Incomplete Block Design in Four Dimensions
}

\author{
Dennis Matundura Mwan*, Mathew Kosgei \\ Email address: \\ denismwan407@gmail.com (D. M. Mwan), Mkosgei12@gmail.com (M. Kosgei) \\ ${ }^{*}$ Corresponding author
}

Department of Statistics and Computer Science, School of Biological and Physical Science, Moi University, Eldoret, Kenya

\section{To cite this article:}

Dennis Matundura Mwan, Mathew Kosgei. Two Combined Alphabetic Optimality Criteria for Second Order Rotatable Designs Constructed Using Balanced Incomplete Block Design in Four Dimensions. International Journal of Data Science and Analysis.

Vol. 4, No. 2, 2018, pp. 32-37. doi: 10.11648/j.ijdsa.20180402.11

Received: May 29, 2018; Accepted: July 6, 2018; Published: August 4, 2018

\begin{abstract}
The theory of optimal experimental designs is concerned with the construction of designs that are optimum with respect to some statistical criteria. Some of these criteria include the alphabetic optimality criteria such as; D-, A-, E-, T-, Gand C- criterion. Compound optimality criteria are those that combine two or more alphabetic optimality criteria. Design that require optimality criteria have specific desired properties that do very well in one design and at the same time perform poorly in another design. Thus, a compound optimality criterion gives a balance to the desirability of any two or more alphabetic optimality criteria. The present paper aims to introduce CD- and DT- criteria which are compound optimality criteria for second order rotatable designs constructed using Balanced Incomplete Block Designs (BIBDs) in four dimensions.
\end{abstract}

Keywords: Optimality Criteria, Compound Criteria, DT-optimum and CD-optimum

\section{Introduction}

Design experts have come to a realization that a design can perform very well in terms of a particular statistical characteristic and still perform poorly in terms of a rival characteristic. In the field of life sciences optimal designs are required in order to cut on cost of experimentation. Kussmaul [15] introduced method that allows for an efficient consideration of nonlinear constraints.

An experimenter is therefore advised to make the choice of a design to be used prior to carrying out any experiment. In statistics, Response Surface Methodology (RSM) explores the relationships between several explanatory variables and one or more response variables. The method was introduced by George E. P. Box and K. B. Wilson [1]. The main idea of RSM is to use a sequence of designed experiments to obtain an optimal response. Box and Wilson [1] suggest using a second-degree polynomial model to do this. They acknowledge that this model is only an approximation, but they use it because such a model is easy to estimate and apply, even when little is known about the process. Statistical approaches such as RSM can be employed to maximize the production of a special substance by optimization of operational factors. In contrast to conventional methods, the interaction among process variables can be determined by statistical techniques [2].

According to Box and Draper [3], RSM is either used to explore response surfaces or to estimate the parameters of a model. Bose and Draper [4] point out that the technique of fitting a response surface is one widely used to aid in the statistical analysis of experimental work in which the response of a product depends in some unknown factors on one or more controllable variables. A particular selection of settings or factor levels at which observations are to be taken is called a design. Designs are usually selected to satisfy some desirable criteria chosen by the experimenter.

The proper meaning of optimal depends on the situation and can include cost effective, minimum variance and minimum bias. Youdim [13] Correctly chosen D-optimum designs provide efficient experimental schemes when the aim of the investigation is to obtain precise estimates of parameters. The commonly used classical optimality criteria 
which were introduced and widely discussed by Pukelsheim [5] includes, Determinant criterion (D-), the average variance criterion (A-), the smallest Eigen value (E-) and the trace criterion (T-). Many results on optimal designs of experiments are derived under the assumption that the statistical model is known at the design stage. Nguyen [14] proposed to use a compound optimality criterion based on the expected population Fisher information matrix in nonlinear mixed effect models. However, rarely it is known in advance which model is the most appropriate. Box and Hunter [6] introduced rotatable designs in order to explore the response surfaces. They developed second order rotatable designs through Schlaflian vectors and matrices. Mylona [12] allowed for a more powerful statistical inference than traditional optimal designs.

According to Draper [7] a second order rotatable design aids the fitting of a second order surface and provides spherical information contours and a third order rotatable design aids the fitting of a third order surface. Thus, the goal of an experiment should be dual: to choose an appropriate design and the most adequate model.

A second degree response model with $k$ factors is represented as follows

$$
w^{\prime}=\left(1, \frac{1}{k}\left(x_{1}^{2}, x_{2}^{2}, x_{3}^{2}, \cdots, x_{k}^{2}\right), x_{1}, x_{2}, x_{3}, \ldots, x_{k}, \frac{1}{\left[\begin{array}{l}
k \\
2
\end{array}\right]}\left(x_{1} x_{2}, x_{1} x_{3}, x_{2} x_{3}, \ldots, x_{k-1} x_{k}\right.\right.
$$

and

$k=$ is the number of factors in the design for this case the factors are four.

\section{Design Matrix}

The generalized design matrix $X$ for the second order rotatable design is given by

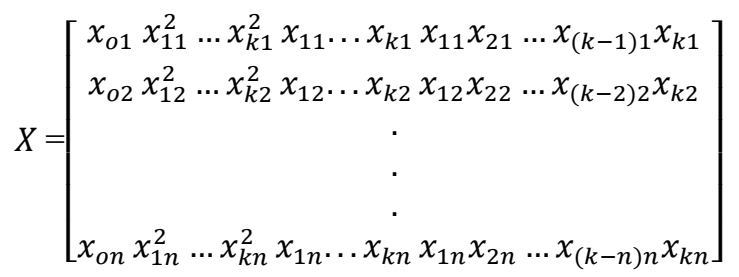

The vector in (3) is partitioned in the following order; the pure quadratic, the linear and the interaction effects. Consequently, the moment matrix is also partitioned as shown below.

$$
M=\left[\begin{array}{ccc}
B & 0 & 0 \\
0 & A_{1} & 0 \\
0 & 0 & A_{2}
\end{array}\right]
$$

where $\beta_{o}$ is the intercept

$\beta_{i}$ is the linear coefficient for the $i^{\text {th }}$ factor $\beta_{i i}$ is the quadratic coefficient for the $i^{\text {th }}$ factors

$\beta_{i j}$ is the cross product coefficient for the $i^{t h}$ and $j^{\text {th }}$ factors $x_{i}$ is the level of the $i^{t h}$ factor

$x_{i} x_{j}$ is the level of the $i^{\text {th }}$ and $j^{\text {th }}$ factor

\section{Evaluation of C-Criterion in Four Dimensions}

The C-criterion for the second order rotatable design in four dimensions is obtained through minimizing the variance of the linear unbiased estimator of the integral function $w^{/}\left(X^{/} X\right)^{-1} w$. This was defined by Elfying [8] as;

$$
C \text { - Criterion }=\iint \ldots \int w^{\prime}\left(X^{/} X\right)^{-1} \mathrm{w} d_{x 1} d_{x 2} \ldots d_{x k} .
$$

where 
where, $M^{-1}=\left[\begin{array}{ccc}\mathrm{B}^{-1} & 0 & 0 \\ 0 & \mathrm{~A}_{1}^{-1} & 0 \\ 0 & 0 & \mathrm{~A}_{2}^{-1}\end{array}\right]$

$$
B^{-1}=\frac{1}{\Delta_{1}}\left[\begin{array}{ccccc}
\alpha & \beta & \beta & \cdots & \beta \\
\beta & \gamma & \mu & \cdots & \mu \\
\beta & \mu & \gamma & \cdots & \mu \\
& & \vdots & & \\
\beta & \mu & \mu & \cdots & \gamma
\end{array}\right]_{[k+1] \times[k+1]}
$$

In which;

$\propto=2(\mathrm{k}+2) \lambda_{4}^{2}, \beta=-2 \lambda_{2} \lambda_{4}, \mu=-\left(\lambda_{4}-\lambda_{2}^{2}\right), \gamma=(\mathrm{k}+1) \lambda_{4}-$ $(\mathrm{k}-1) \lambda_{2}^{2}$ and

$$
\begin{gathered}
\Delta_{1}=2\left[(\mathrm{k}+2) \lambda_{4}^{2}-\mathrm{k} \lambda_{2}^{2} \lambda_{4}\right] ; \\
A_{1}^{-1}=\frac{1}{\lambda_{2}}\left[\begin{array}{ccccc}
1 & 0 & 0 & \cdots & 0 \\
0 & 1 & 0 & \cdots & 0 \\
0 & 0 & 1 & \cdots & 0 \\
0 & 0 & 0 & \cdots & 1
\end{array}\right]_{[K] X[K],}
\end{gathered}
$$

and$$
A_{2}^{-1}=\frac{1}{\lambda_{4}}\left[\begin{array}{ccccc}
1 & 0 & 0 & \cdots & 0 \\
0 & 1 & 0 & \cdots & 0 \\
0 & 0 & 1 & \cdots & 0 \\
0 & & & \ddots & \\
0 & 0 & 0 & \cdots & 1
\end{array}\right]_{\left[\frac{k}{2}\right] \times\left[\frac{k}{2}\right]}
$$

The coefficient matrix $K^{\prime}$ is determined from a reduced parameters system, where the reduced pure quadratic and the interaction effect is that;

$$
K^{\prime} \beta=\left[\begin{array}{c}
\beta_{0} \\
\frac{\sum_{i=1}^{k} \beta_{i i}}{k} \\
\beta_{i}^{k} \\
\sum_{i<j=1}^{k} \beta_{i j} \\
\left(\begin{array}{l}
k \\
2
\end{array}\right) \\
i=1,2, \ldots, k
\end{array}\right]
$$

Where

$$
\beta=\left(\beta_{0}, \beta_{11}, \beta_{22}, \ldots, \beta_{k k}, \beta_{1}, \beta_{2}, \ldots, \beta_{k}, \beta_{12}, \ldots, \beta_{k-1, k}\right)^{\prime}
$$

is the full parameter system and is the coefficient of the second order model

$$
y=\beta_{0}+\beta_{11} x_{1 u}{ }^{2}+\beta_{22} x_{2 u}{ }^{2}+\ldots+\beta_{k k} x_{k u}{ }^{2}+\beta_{1} x_{1 u}+\beta_{2} x_{2 u}+\ldots \beta_{k} x_{k u}+\beta_{12} x_{1 u} x_{2 u}+\ldots+\beta_{k-1, k} x_{k-1, u}, x_{k u} \text { and } K^{\prime} \text { defined as }
$$

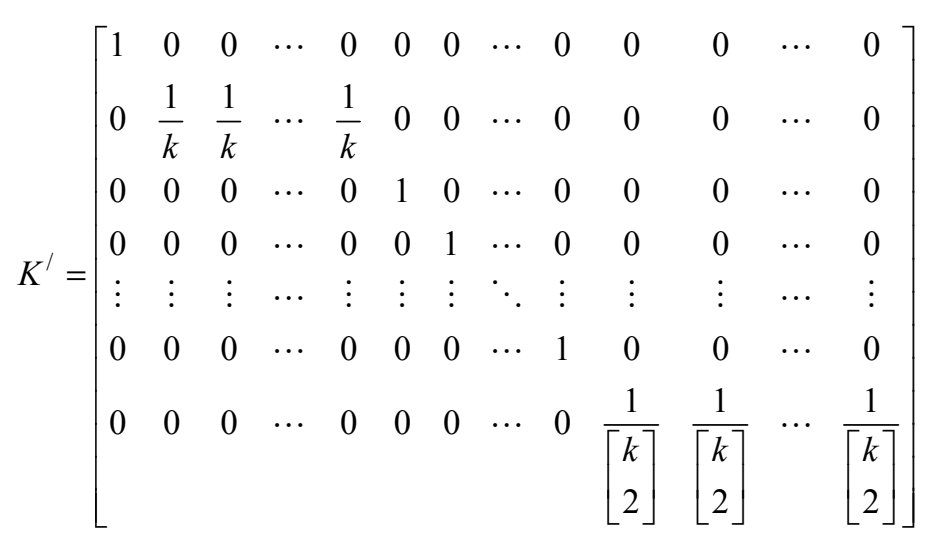

is generalized coefficient matrix of the parameter system of interest.

The coefficients of $\mathrm{w}^{\prime}$ in (3) are the diagonal elements of a $\mathrm{k}$ matrix in the parameter system of interest.

\section{Information Matrix}

Mwan and Rambaei [9] used the moment matrix for second order model to determine the information matrix for the parameter system of interest. Its information matrix $\mathrm{C}$ is determined by

$$
C_{k}(M)=\left[K_{k}^{\prime} M_{k}^{-1} K_{k}\right]^{-1}
$$

where $M=\frac{1}{N} x^{\prime} x$ and $k$ is the number of factors and $X$ is as defined in (4)

$$
C_{k}(M)
$$




$$
\left.=\left[\begin{array}{cccccccc}
1 & k \lambda_{2} & 0 & 0 & 0 & \cdots & \cdots & 0 \\
k \lambda_{2} & k[k+2] \lambda_{4} & 0 & 0 & 0 & \cdots & \cdots & 0 \\
0 & 0 & \lambda_{2} & 0 & 0 & \cdots & \cdots & 0 \\
0 & 0 & 0 & \lambda_{2} & 0 & \cdots & \cdots & 0 \\
0 & 0 & 0 & 0 & \lambda_{2} & \cdots & \cdots & 0 \\
\vdots & \vdots & \vdots & \vdots & \vdots & \ddots & \vdots & \vdots \\
\vdots & \vdots & \vdots & \vdots & \vdots & \vdots & \ddots & \vdots \\
0 & 0 & 0 & 0 & 0 & 0 & 0 & {[k} \\
2
\end{array}\right] \lambda_{4}\right]_{[k+3][k+3]}
$$

Using the elements of the inverse of the moment matrix in (7), (8) and (9) respectively (3) is obtained.

The computation for the C- criterion was portioned into three parts; the linear effects the pure quadratic and the interaction effects which were denoted as $\beta_{\mathrm{ij}}$. For the 64 points the parts are $\beta_{11}, \beta_{12}$ and $\beta_{13}$ with the help of matlab software.

\section{5. $D$ - Criterion for $2^{\text {nd }}$ Degree Design with Sixty Four Points}

For $k=4$ factors, the information matrix is given as;

$$
C_{4}(M)=\left[\begin{array}{ccccccc}
1 & 4 \lambda_{2} & 0 & 0 & 0 & 0 & 0 \\
4 \lambda_{2} & 24 \lambda_{4} & 0 & 0 & 0 & 0 & 0 \\
0 & 0 & \lambda_{2} & 0 & 0 & 0 & 0 \\
0 & 0 & 0 & \lambda_{2} & 0 & 0 & 0 \\
0 & 0 & 0 & 0 & \lambda_{2} & 0 & 0 \\
0 & 0 & 0 & 0 & 0 & \lambda_{2} & 0 \\
0 & 0 & 0 & 0 & 0 & 0 & 6 \lambda_{4}
\end{array}\right]
$$

Thus the determinant criterion is

$$
\phi_{0} C_{4}(M)=\left[\operatorname{Det}_{4}(M)\right]^{\frac{1}{7}}=\left[24 \lambda_{2}{ }^{4} \lambda_{4}\left[6 \lambda_{4}-4 \lambda_{2}^{2}\right]\right]^{\frac{1}{7}}
$$

Now from (13), we have, for the designs with $k=4$, we substitute the following to (14)

$$
\lambda_{2}=0.233258 \rho^{2} \text { and } \lambda_{4}=0.06251 \rho^{4}
$$

Thus,

$$
\emptyset_{0} C_{4}(M)=\left[24 \lambda_{2}^{4} \lambda_{4}\left(6 \lambda_{4}-4 \lambda_{2}^{2}\right)\right]^{1 / 7}=0.3541807443 .(16)
$$

$$
w^{\prime[4]}=\left[1, \frac{1}{4} x_{1}^{2}, \frac{1}{4} x_{2}^{2}, \frac{1}{4} x_{3}^{2}, \frac{1}{4} x_{4}^{2}, x_{1}, x_{2}, x_{3}, x_{4}, \frac{1}{6} x_{1} x_{2}, \frac{1}{6} x_{1} x_{3}, \frac{1}{6} x_{1} x_{4}, \frac{1}{6} x_{2} x_{3}, \frac{1}{6} x_{2} x_{4}, \frac{1}{6} x_{3} x_{4}\right]
$$

Taking only the pure quadratic terms from (21) then we have;

$$
\begin{gathered}
w^{\prime[3]}=\left[1, \frac{1}{4} x_{1}^{2}, \frac{1}{4} x_{2}^{2}, \frac{1}{4} x_{3}^{2}, \frac{1}{4} x_{4}^{2}\right] \\
=[1 \\
\left.\frac{1}{4} x_{1}^{2} \frac{1}{4} x_{2}^{2} \frac{1}{4} x_{3}^{2} \frac{1}{4} x_{4}^{2}\right]
\end{gathered}
$$

\section{6. $T$ - Criterion for $2^{\text {nd }}$ Degree Design with Sixty Four Points}

The trace criterion is given as;

$$
\phi_{1} C_{4}(M)=\frac{1}{7} \operatorname{trace}_{4}(M)
$$

where

$$
C_{4}(M)=\left[\begin{array}{ccccccc}
1 & 4 \lambda_{2} & 0 & 0 & 0 & 0 & 0 \\
4 \lambda_{2} & 24 \lambda_{4} & 0 & 0 & 0 & 0 & 0 \\
0 & 0 & \lambda_{2} & 0 & 0 & 0 & 0 \\
0 & 0 & 0 & \lambda_{2} & 0 & 0 & 0 \\
0 & 0 & 0 & 0 & \lambda_{2} & 0 & 0 \\
0 & 0 & 0 & 0 & 0 & \lambda_{2} & 0 \\
0 & 0 & 0 & 0 & 0 & 0 & 6 \lambda_{4}
\end{array}\right]
$$

Thus,

$\varphi_{1} C_{4}=\frac{1}{7}\left[1+24 \lambda_{4}+4 \lambda_{2}+6 \lambda_{4}\right]$ and by further simplification we get;

$$
\phi_{1} C_{4}=\frac{1}{7}\left[1+4 \lambda_{2}+30 \lambda_{4}\right]
$$

Now from (17), we have, for the designs with $k=4$,

Substituting for these values of $\lambda_{2}$ and $\lambda_{4}$ given in (15) then;

The Trace criterion is

$$
\emptyset_{1} \mathrm{C}_{4}=0.54440474286 .
$$

\section{C-Criterion for $2^{\text {nd }}$ Degree Design with Sixty Four Points}

Substituting $\lambda_{2}$ and $\lambda_{4}$ given in (15) to (7) yields the information matrix given as;

$$
B^{-1}=\left[\begin{array}{c}
2.3825-1.4817-1.4817-1.4817-1.4817 \\
-1.48177 .5871-0.4116-0.41160 .4116 \\
-1.4817-0.41167 .5871-0.41160 .4116 \\
-1.4817-0.4116-0.41167 .5871-0.4116 \\
-1.4817-0.4116-0.4116-0.41167 .5871
\end{array}\right] \text {, }
$$

The vector $w$ expanded to include all terms of a second order rotatable design in four dimensions was given by,

$$
\left[\begin{array}{l}
2.3825-1.4817-1.4817-1.4817-1.4817 \\
-1.48177 .5871-0.4116-0.4116-0.4116 \\
-1.4817-0.41167 .5871-0.4116-0.4116 \\
-1.4817-0.4116-0.41167 .5871-0.4116 \\
-1.4817-0.4116-0.4116-0.41167 .5871
\end{array}\right]\left[\begin{array}{l}
1 \\
\frac{1}{4} x_{1}^{2} \\
\frac{1}{4} x_{2}^{2} \\
\frac{1}{4} x_{3}^{2} \\
\frac{1}{4} x_{4}^{2}
\end{array}\right],
$$


Substituting (20) and (22) to integral function in (2) gives;

$$
\begin{gathered}
\iiint \int_{-1}^{1}\left[2.3825-0.3704 x_{1}^{2}-0.3704 x_{2}^{2}-0.3704 x_{3}^{2}-\right. \\
0.3704 x_{4}^{2}-0.3704 x_{1}^{2}+0.4742-0.02573 x_{1}^{2} x_{2}^{2}- \\
0.02573 x_{1}^{2} x_{3}^{2}-0.02573 x_{1}^{2} x_{4}^{2}-0.3704 x_{2}^{2}- \\
0.02573 x_{2}^{2} x_{1}^{2}+0.4742 x_{2}^{4}-0.02573 x_{2}^{2} x_{3}^{2}- \\
0.02573 x_{2}^{2} x_{4}^{2}-0.3704 x_{3}^{2}-0.02573 \\
x_{1}^{2} x_{3}^{2}-0.02573 x_{2}^{2} x_{3}^{2}+0.4742 x_{3}^{4}-0.02573 x_{3}^{2} x_{4}^{2}- \\
0.3704 x_{4}^{2}-0.02573 x_{1}^{2} x_{4}^{2}-0.02573 x_{2}^{2} x_{4}^{2}- \\
\left.0.02573 x_{3}^{2} x_{4}^{2}+0.4742 x_{4}^{4}\right] d_{x 1} d_{x 2} d_{x 3} d_{x 4} \\
\beta_{11}=3.8586 .
\end{gathered}
$$

Again by Substituting $\lambda_{2}$ given in (15) to (8) gives

$$
A_{1}^{-1}=\left[\begin{array}{cccc}
4.2871 & 0 & 0 & 0 \\
0 & 4.2871 & 0 & 0 \\
0 & 0 & 4.2871 & 0 \\
0 & 0 & 0 & 4.2871
\end{array}\right]
$$

Taking only the linear terms in (21) the outcome is;

$$
w^{\prime[4]}=\left[x_{1}, x_{2}, x_{3}, x_{4}\right]
$$

Substituting sub matrix in (24) and the linear terms in (25) to the integral function in (2) gives;

$$
\begin{gathered}
\iiint \int_{-1}^{1}\left[4.2871 x_{1}^{2}+4.2871 x_{2}^{2}\right. \\
\left.+4.2871 x_{3}^{2}+4.2871 x_{4}^{2}\right] d_{x 1} d_{x 2} d_{x 3} d_{x 4} \\
\beta_{12}=11.43232 .
\end{gathered}
$$

Substituting $\lambda_{4}$ given in (15) to (9) gives

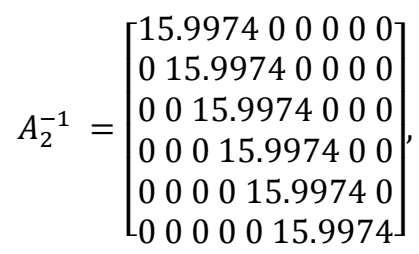

Taking only the interactions terms of vector $w$ in (21) we have;

$$
w^{\prime[3]}=\left[\frac{1}{6} x_{1} x_{2}, \frac{1}{6} x_{1} x_{3}, \frac{1}{6} x_{1} x_{4}, \frac{1}{6} x_{2} x_{3}, \frac{1}{6} x_{2} x_{4}, \frac{1}{6} x_{3} x_{4}\right]
$$

Substituting (27) and (28) to the integral function in (2) yields;

$$
\begin{gathered}
\iiint \int_{-1}^{1}\left[15.9974 x_{1}^{2} x_{2}^{2}+15.9974 x_{1}^{2} x_{3}^{2}+15.9974 x_{1}^{2} x_{4}^{2}\right. \\
+15.9974 x_{2}^{2} x_{3}^{2}+15.9974 x_{2}^{2} x_{4}^{2} \\
\left.+15.9974 x_{3}^{2} x_{4}^{2}\right] d_{x 1} d_{x 2} d_{x 3} d_{x 4} \\
\beta_{13}=21.33
\end{gathered}
$$

The C-criterion for a design with 64 points is;

$$
\beta_{11}+\beta_{12}+\beta_{13}=36.62092
$$

\section{DT- Optimality}

This paper combines two alphabetic optimality criteria Dand $\mathrm{T}$ - by using the concept that was introduced by Atkinson [10], where DT optimality criterion is a combination of Doptimality criterion for parameter estimation with the $\mathrm{T}$ optimality criterion for discriminating between models. The DT- criterion provides a specified balance between model discrimination and parameter estimation.

The Generalized Determinant and Trace Criteria are given as;

$$
\begin{aligned}
& \phi_{0} C_{k}(M)=\left[k\left[\begin{array}{l}
k \\
2
\end{array}\right] \lambda_{2}{ }^{k} \lambda_{4}\left[[k+2] \lambda_{4}-k \lambda_{2}{ }^{2}\right]\right]^{\frac{1}{k+3}} \\
& \phi_{1} C_{k}(M)=\frac{1}{k+3}\left[1+(k+2) k \lambda_{4}+k \lambda_{2}+\left(\begin{array}{l}
k \\
2
\end{array}\right) \lambda_{4}\right]
\end{aligned}
$$

The DT-criterion is given by the formula;

$$
\emptyset_{2}^{D T}(\varepsilon)=(1-\mathrm{k}) \log \Delta_{1}(\varepsilon)+\left(\frac{k}{p_{1}}\right) \log \left|m_{1}(\varepsilon)\right| .
$$

where $\emptyset_{2}^{D T}(\varepsilon)$ is a convex combination of two design criteria, the first criterion is $\log \Delta_{1}(\varepsilon)$ which is the logarithm of Toptimality and the second $\log \left|m_{1}(\varepsilon)\right|$ is also the logarithm of D- optimality.

Designs maximizing (33) are called DT-optimum. The quantities in (31) and (32) are substituted in (33) to obtain the DT-optimality criterion.

For $\mathrm{k}=4$, the determinant criterion is given in (16) and the trace criterion in (19) substituting it in the compound formula given in (33) results to the DT-compound optimality criteria is;

Whence,

$$
\begin{gathered}
\emptyset_{1}^{\mathrm{DT}}(\varepsilon)=(1-\mathrm{k}) \log 0.5440474286 \\
+\left(\frac{\mathrm{k}}{\mathrm{p}_{1}}\right) \log 0.3541807443 \\
=(1-4) \log 0.5440474286+\frac{4}{7} \log (0.3541807443) \\
=0.7930897141-0.257585745 \\
\emptyset_{2}^{D T}(\varepsilon)=0.5355039691 .
\end{gathered}
$$

\section{CD-Criterion for 64 Points in Four Dimension}

The CD-optimality that combines C-optimality for a model selection and D-optimality for parameter estimation which was introduced by Atkinson [11], provides a specified balance between model discrimination and parameter estimation too. The criterion to be maximized was;

$$
\emptyset_{3}^{C D}(\varepsilon)=\left(\frac{k}{p_{1}}\right) \log \left|m_{1}(\varepsilon)\right|-(1-\mathrm{k}) \log w^{T} M^{-1}(\varepsilon) w .
$$

where $\emptyset_{3}^{C D}(\varepsilon)$ is a convex combination of two design criteria, the first criterion is $\log \left|m_{1}(\varepsilon)\right|$ which is the logarithm of D- 
optimality and the second $\log w^{T} M^{-1}(\varepsilon) w$ is the logarithm of C- optimality.

The designs maximizing (35) are called CD-optimality. The quantities in (2) and (31) were substituted in (35) to obtain the CD-optimality criterion.

The Determinant criterion was given in (16) and the $\mathrm{C}$ criterion in (30) for $\mathrm{k}=4$ using the compound formula stated in (35) gave the CD- compound optimality criterion as;

$$
\begin{aligned}
& \emptyset_{1}^{C D}(\varepsilon)=\frac{4}{7} \log 0.3541807443+3 \log 36.62092 \\
& \emptyset_{1}^{C D}(\varepsilon)=-0.257585745+4.691187752 \\
& \emptyset_{1}^{C D}(\varepsilon)=4.4336 .
\end{aligned}
$$

\section{Conclusion}

The study concludes by combining D- and T-optimality to get DT-(compound optimality). The design under consideration is said to be better than the alphabetic optimality design in four factors constructed by Mwan, kosgei and Rambaei [9]. The D-, T- and DT- optimality criteria are compared and there is a clear balance brought by the DTcombination. Again the analysis of the two alphabetic criteria and the compound criterion above show that design experts will prefer characteristics from the D- optimality criteria. However, when the experiment requires the utilization of the two properties the compound optimality serves the deal. This is from the result obtained above for the $\mathrm{D}$ - criterion the value was 0.3541807443 , the T- criterion become 0.5440474286 but the combination of the two gave a value of 0.5355039691 which is in between the two criteria. Hence, a balance between parameter estimation and model discrimination is achieved. Again, the result obtained above for the D- criterion the value was 0.3541807443 , the C- criterion become 36.62092 but the combination of the two gave a more homogenous value tending to zero 4.4336 as compared to single optimality criterion. This clearly brought a balance between parameter estimation and model discrimination.

\section{References}

[1] Box, G. and K. Wilson, on the experimental attainment of optimum conditions, in Breakthroughs in statistics. 1992, Springer. p. 270-310.
[2] Asadi, H., et al., Robust optimal motion cueing algorithm based on the linear quadratic regulator method and a genetic algorithm. IEEE Transactions on Systems, Man, and Cybernetics: Systems, 2017. 47(2): p. 238-254.

[3] Box, G. and N. Draper, A basis for the selection of a response surface design. Journal of the American Statistical Association, 1959. 54(287): p. 622-654.

[4] Bose, R. and N. Draper, Second order rotatable designs in three dimensions. The Annals of Mathematical Statistics, 1959: p. 1097-1112.

[5] Pukelsheim, F., Optimal design of experiments. Vol. 50. 1993: siam.

[6] Box, G. and J. Hunter, Multi-factor experimental designs for exploring response surfaces. The Annals of Mathematical Statistics, 1957: p. 195-241.

[7] Draper, N. R., Second order rotatable designs in four or more dimensions. The Annals of Mathematical Statistics, 1960. 31(1): p. 23-33.

[8] Elfving, G., Optimum allocation in linear regression theory. The Annals of Mathematical Statistics, 1952. 23(2): p. 255-262.

[9] Mwan, D., M. Kosgei, and S. Rambaei, DT-optimality Criteria for Second Order Rotatable Designs Constructed Using Balanced Incomplete Block Design.

[10] Atkinson, A. C., DT-optimum designs for model discrimination and parameter estimation. Journal of Statistical planning and Inference, 2008. 138(1): p. 56-64.

[11] Atkinson, A., A. Donev, and R. Tobias, Optimum experimental designs, with SAS, vol. 34 of Oxford Statistical Science Series. 2007, Oxford University Press Oxford, UK.

[12] Mylona, K., Goos, P., \& Jones, B. (2014). Optimal design of blocked and split-plot experiments for fixed effects and variance component estimation. Technometrics, 56(2), 132-144.

[13] Youdim, K., Atkinson, A. C., Patan, M., Bogacka, B., \& Johnson, P. (2010). Potential Application of D-Optimum Designs in the Efficient Investigation of Cytochrome P450 Inhibition Kinetic Models. Drug metabolism and disposition, dmd-11.

[14] Nguyen, T. T., Bénech, H., Delaforge, M., \& Lenuzza, N. (2016). Design optimisation for pharmacokinetic modeling of a cocktail of phenotyping drugs. Pharmaceutical statistics.

[15] Kussmaul, R., Zogg, M., \& Ermanni, P. (2018). An optimality criteria-based algorithm for efficient design optimization of laminated composites using concurrent resizing and scaling. Structural and Multidisciplinary Optimization, 1-16. 